\title{
Electron Energy Loss Spectroscopy of Actinides at the Nanogram Scale
}

Alexander Müller ${ }^{1,2}$, Gauthier J.-P. Deblonde ${ }^{3}$, Peter Ercius ${ }^{1}$, Rebecca J. Abergel ${ }^{3,4}$ and Andrew M. Minor $^{1,2}$

1. National Center for Electron Microscopy, Molecular Foundry, Lawrence Berkeley National Laboratory, Berkeley, CA 94720, USA

2. Department of Materials Science and Engineering, University of California, Berkeley, CA 94720, USA

3. Chemical Sciences Division, Lawrence Berkeley National Laboratory, Berkeley, CA 94720, USA

4. Department of Nuclear Engineering, University of California, Berkeley, CA 94720, USA

Actinide atoms have seven valence 5f-orbitals, leading to complicated electronic structures and fascinating properties for both free metal ions and their compounds [1]. Yet, comparatively little is known about most of them, mostly because they are without exception radioactive and handling them poses an inherent health risk. Due to contamination and radiation safety issues, it is often necessary to extensively prepare experiments, perform dry runs using non-radioactive surrogates, and to use dedicated instruments and facilities. Most actinides are synthetic elements and their preparation is timeconsuming, difficult and therefore expensive, with microgram prices reaching thousands of dollars.

Of the berkelium isotopes, ${ }^{249} \mathrm{Bk}$ is the only one that can be produced and purified in reasonable amounts (1-5 mg/year). Nonetheless, it is rarely produced because it requires a dedicated nuclear reactor and campaign. ${ }^{249} \mathrm{Bk} \beta$-decays into ${ }^{249} \mathrm{Cf}$ with a half-life of 330 days, and because of this short half-life relatively little is known about its properties. For example, only in the last two years were single crystal structures of Bk(III) compounds reported and the +IV valence state stabilized in solution [2,3]. Notably, we could not find electron energy loss spectra (EELS), even though both EELS and transmission electron microscopy (TEM) in general have proven useful for analyzing radioactive materials [4]. Particularly for actinides, which are often only available in small amounts and self-contaminate due to radioactive decay, several advantages of electron microscopy for nano-sampling are evident [5]. Here, we focus on EELS, which can give insight into the band structure using only very small sample amounts, and discuss the example of Bk.

Solid $\mathrm{BkCl}_{3}$ was obtained from the Oak Ridge National Laboratory and a $0.1 \mathrm{M}$ stock solution was prepared with $\mathrm{HCl}$. This stock solution was diluted with deionized water so that each microliter contained approximately $10 \mathrm{ng}$ of $\mathrm{Bk}^{3+}$. As radioactive decay rates are well documented, the concentration of the actinide could be calculated directly from decay measurement. A drop of $1 \mu 1$ was then deposited onto a TEM grid with an ultrathin carbon film using an air displacement pipette and left to dry. Photographs taken both of the initial drop and of the dried deposit show that the drop contracted evenly. As the drop contracts and its volume decreases, the saturation of the solved species goes up and the solution becomes supersaturated, leading to precipitation of the $\mathrm{BkCl}_{3}$ in a much smaller area of the TEM grid than the initial drop. As the dry sample is non-dispersible and has an activity of only $15.8 \mu \mathrm{Ci}$, it can be handled without shielding and with minimal safety precautions at the TEM.

All samples were analysed in the TEAM 0.5 microscope, a double-corrected FEI Titan with EELS acquired with an energy resolution of $\sim 0.9 \mathrm{eV}$. The microscope was operated in the high-angle annular dark-field (HAADF) scanning TEM (STEM) mode, in which heavy elements scatter more than light 
elements and in which $\mathrm{BkCl}_{3}$ appeared very bright. The sample was contaminated with $\mathrm{NaCl}$ and $\mathrm{Fe} / \mathrm{Cr}-$ containing compounds, presumably stemming from the process by which the radioelements were separated. The heavily-scattering $\mathrm{BkCl}_{3}$ could easily be distinguished from contaminations and was found within a few minutes, indicating good coverage. We estimate that we could reduce the material amount by several orders of magnitude and still easily find the sample. $\mathrm{BkCl}_{3}$ crystallized into nanoparticles with a diameter below $5 \mathrm{~nm}$ that in turn formed large agglomerations (Figure 1a). The onset of the $\mathrm{O}_{4,5}$-edge was $100 \mathrm{eV}$, of the $\mathrm{N}_{4,5}$-edge $888 \mathrm{eV}$ (Figure 1b), and of the $\mathrm{M}_{4,5}$-edge $4120 \mathrm{eV}$ (Figure 1c). The number and shapes of the edges are still under discussion, and awaiting measurements of the decay product ${ }^{249} \mathrm{Cf}$ which probably contributes to the spectra.

The approach described here is uniquely suited for the safe study of compounds of actinides, especially when only available in small amounts, and should be directly applicable to compounds of several other elements. Actinium $\left({ }^{227} \mathrm{Ac}\right.$, half-life: 22 years $)$, protactinium $\left({ }^{231} \mathrm{~Pa}\right.$, half-life: 32,760 years $)$, californium $\left({ }^{249} \mathrm{Cf}\right.$, half-life: 351 years $)$, einsteinium $\left({ }^{252} \mathrm{Es}\right.$, half-life: 471 days $)$, fermium $\left({ }^{257} \mathrm{Fm}\right.$, half-life: 100 days $)$, and mendelevium $\left({ }^{258} \mathrm{Md}\right.$, half-life: 51 days) are all very scarce and have been only rarely investigated as of now, but have half-lives sufficiently long for an in-depth characterization. We believe that TEM, and especially EELS, can significantly further the study of actinide compounds, and will in our presentation give a detailed discussion of this approach.

\section{References:}

[1] KT Moore and G van der Laan, Reviews of Modern Physics 81 (2009), p. 235.

[2] GJP Deblonde, M Sturzbecher-Hoehne, PB Rupert, DD An, MC Illy, CY Ralston, J Brabec, WA de Jong, RK Strong and RJ Abergel, Nature Chemistry 9 (2017), p. 843.

[3] MA Silver, SK Cary, JA Johnson, RE Baumbach, AA Arico, M Luckey, M Urban, JC Wang, MJ Polinski, A Chemey, G Liu, K-W Chen, SM Van Cleve, ML Marsh, TM Eaton, LJ van de Burgt, AL Gray, DE Hobart, K Hanson, L Maron, F Gendron, J Autschbach, M Speldrich, P Kögerler, P Yang, J Braley and TE Albrecht-Schmitt, Science 353 (2006), p. 889.

[4] KT Moore, Micron 41 (2010), p. 336.

[5] EC Buck and JA Fortner, Ultramicroscopy 67 (1996), p. 69.

[6] Procurement of ${ }^{249} \mathrm{Bk}$, sample preparation, and work at the Molecular Foundry were supported by the Office of Science, Office of Basic Energy Sciences, of the U.S. Department of Energy under Contract No. DE-AC02-05CH11231.
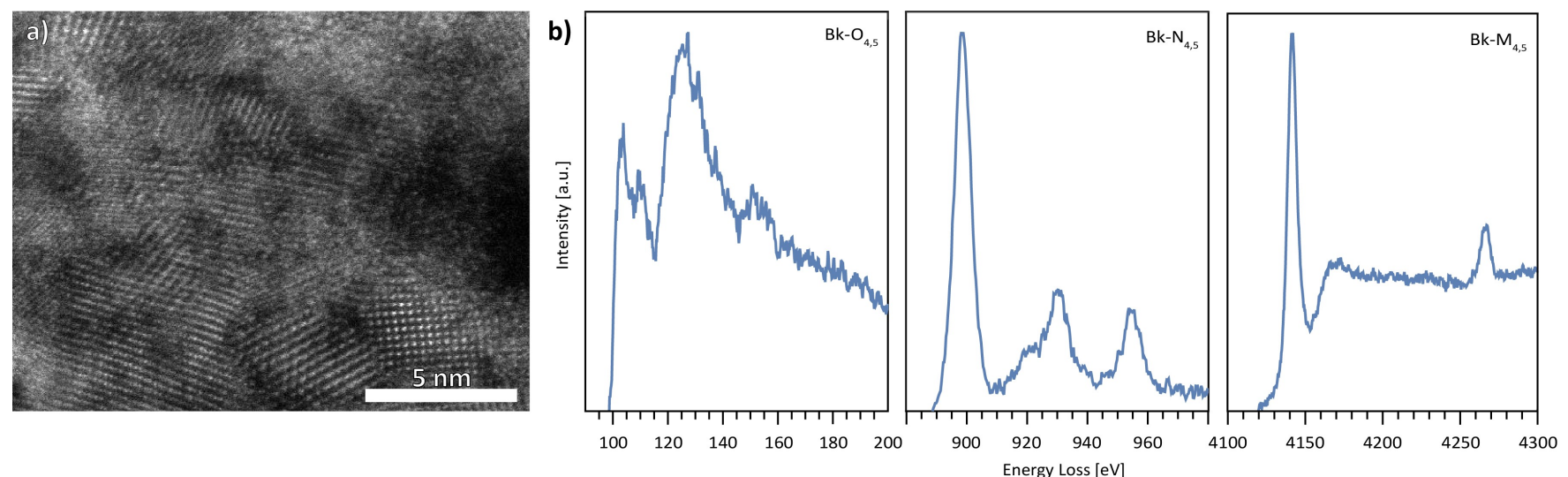

Figure 1. (a) HAADF-STEM image of several nanoparticles; (b) $\mathrm{O}_{4,5^{-}}, \mathrm{N}_{4,5^{-}}$and $\mathrm{M}_{4,5}$-edge of $\mathrm{Bk}^{3+}$. 